\title{
Changes in $\lg G$ and total plasma protein glycomes in acute systemic inflammation
}

SUBJECT AREAS:

GLYCOBIOLOGY

BIOMARKER RESEARCH

Received

28 November 2013

Accepted

24 February 2014

Published

11 March 2014

Correspondence and requests for materials should be addressed to

O.G. Jogornik@ pharma.hr) or G.L. (glauc@pharma.hr)

* These authors contributed equally to this work.

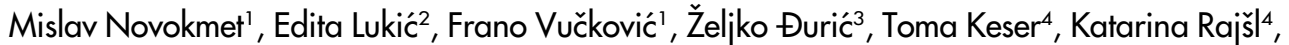 \\ Daniel Remondini ${ }^{5}$, Gastone Castellani ${ }^{5}$, Hrvoje Gašparović ${ }^{3}$, Olga Gornik ${ }^{4 *} \&$ Gordan Lauc ${ }^{1,4 *}$
}

\begin{abstract}
${ }^{1}$ Genos Glycoscience Laboratory, Zagreb, Croatia, ${ }^{2}$ Department of Orthopaedic Surgery University Hospital Centre Zagreb, Croatia, ${ }^{3}$ Clinic for Cardiac Surgery, University Hospital Centre Zagreb, Croatia, ${ }^{4}$ University of Zagreb, Faculty of Pharmacy and Biochemistry, Department of Biochemistry and Molecular Biology, Zagreb, Croatia, ${ }^{5}$ Department of Physics and Astronomy DIFA, University of Bologna, Bologna, Italy.
\end{abstract}

Recovery after cardiac surgery is a complex process that has to compensate for both individual variability and extensive tissue damage in the context of systemic inflammation. Protein glycosylation is essential in many steps of the inflammatory cascade, but due to technological limitations the role of individual variation in glycosylation in systemic inflammation has not been addressed until now. We analysed composition of the total plasma and IgG $\mathbf{N}$-glycomes in 107 patients undergoing cardiac surgery. In nearly all individuals plasma $\mathrm{N}$-glycome underwent the same pattern of changes in the first $72 \mathrm{~h}$, revealing a general mechanism of glycosylation changes. To the contrary, changes in the IgG glycome were very individualized.

Bi-clustering analysis revealed the existence of four distinct patterns of changes. One of them, characterized by a rapid increase in galactosylated glycoforms, was associated with nearly double mortality risk measured by EuroSCORE II. Our results indicate that individual variation in IgG glycosylation changes during acute systemic inflammation associates with increased mortality risk and indicates new avenues for the development of personalized diagnostic and therapeutic approach.

$\Lambda$ -glycosylation of human proteins is an essential posttranslational modification ${ }^{1}$ generated by a complex biosynthetic pathway comprising hundreds of glycosyltransferases, glycosidases, transcriptional factors, ion channels and other proteins ${ }^{2}$. This process results in the creation of branched oligosaccharide chains, called glycans, which become integral part of proteins and significantly contribute to their structure and function ${ }^{3}$.

Structural details of the attached glycans are of great physiological significance and many pathological conditions are associated with various types of glycan changes ${ }^{4,5}$. Glycosylation appears to be particularly important in the immune system ${ }^{6}$ and immunoglobulin $\mathrm{G}(\mathrm{IgG})$ is one of the best-studied glycoproteins. Each $\mathrm{C}_{\mathrm{H}} 2$ domain of the Fc region of IgG heavy chains carries a covalently attached bi-antennary $N$-glycan at the highly conserved asparagine 297 residue $^{7}$. These glycans are essential for all pro-inflammatory activities of antibodies by maintaining the heavy chains in an open conformation required for Fc $\gamma \mathrm{R}$ interactions and even a small change in their composition has dramatic consequences for effector function of $\operatorname{IgG}^{8,9}$. The addition of fucose to the core of these glycans radically reduces IgG binding to $\mathrm{Fc} \gamma \mathrm{RIII}^{10,11}$ preventing initiation of antibody dependent cellular cytotoxicity (ADCC) $)^{12,13}$ and destruction of target cells. On the other hand, the addition of sialic acid converts IgG from pro-inflammatory to anti-inflammatory agent ${ }^{14,15}$. Instead of binding to Fc $\gamma$ Rs, sialylated Fcs initiate an anti-inflammatory cascade involving the lectin receptor DC-SIGN ${ }^{16,17}$. This leads to upregulated surface expression of the inhibitory Fc $\gamma$ RIIb on inflammatory cells, thus attenuating autoantibody-initiated inflammation. Sialylation of IgG was also found to be essential for the function of intravenous immunoglobulin (IVIG) ${ }^{18}$, a therapeutic preparation of highly purified polyclonal IgG antibodies widely used for the treatment of a number of autoimmune diseases.

The importance of individual variation in glycosylation in acute inflammation has not been extensively studied, primarily due to the absence of reliable quantitative methods for high-throughput glycomics. We have recently developed methods for quantitative analysis of plasma ${ }^{19}$ and $\operatorname{IgG}^{20}$ glycomes and here we have applied them to follow the dynamics of glycosylation changes during early course of systemic inflammation caused by cardiac surgery. Acute systemic inflammation is a part of many pathological events and patient's inflammatory response often determines outcome of a disease. Controlling the inflammatory cascade still represents a great challenge, due to its complexity and individual physiological differences. Cardiac surgery is a procedure that provokes a vigorous inflammatory response, which has important clinical implications $\mathrm{s}^{21-23}$. This inflammatory 
response is caused by blood contact with foreign surfaces and the activation of complement. Besides the activation of the complement system, increasing production of cytokines, oxygen radicals, release of endothelin and the expression of adhesion molecules on leukocytes and the endothelium represent main molecular mechanisms of such inflammation. Cardiovascular surgery with cardiopulmonary bypass (CPB) has improved in past decades, but inflammatory activation in this setting is still unpredictable and is associated with several postoperative complications ${ }^{24}$. It is generally accepted that $\mathrm{CPB}$ initiates a whole-body inflammatory reaction. Factors influencing incidence, severity, and clinical outcome of the inflammatory response, and in particular the reasons why certain patients develop life-threatening perioperative complications, are currently not well understood.

EUROSCORE (European System for Cardiac Operative Risk Evaluation) is a risk model which allows the calculation of the risk of death after a heart operation. The model asks for 17 items of information about the patient, the state of the heart and the proposed operation $^{25}$, and uses logistic regression to calculate the risk of death $^{26}$. This model has been adopted worldwide, becoming the most widely used risk index for cardiac surgery ${ }^{27}$, and its use is believed to have contributed substantially to the improvement in the results of heart surgery seen at the beginning of the millennium.

Due to enhanced production of cytokines in cardiac patients compared to other surgical procedures and modulatory effects of IVIG on the cytokine network, the supplemental application of immunoglobulins represents a potential therapeutic concept in cardiac postoperative high risk patients ${ }^{21}$. From the results of different studies it seems that immunoglobulins are unlikely to improve outcome in the entire group of patients, but only in specific subgroups. There is a possibility that dynamics and potential individuality of glycosylation changes analyzed in this work should be considered when the immunomodulation therapies are subjected.

\section{Results}

Glycosylation of plasma proteins. Total plasma $N$-glycome has been analysed in 107 individuals (Table 1) at three time-points: (1) prior to surgical procedure (day 0), (2) on the first postoperative day (day 1) and (3) on the third postoperative day (day 3). N-linked glycans were released from plasma samples using PNGase F and separated into 16 chromatographic peaks (Figure 1) as we have described previously ${ }^{19}$. Since all patients have received heparin during the surgical procedure, the first six glycan peaks that showed aberrant chromatogram pattern and presumably contained degradation products from heparin have been excluded from the glycome for quantitative analysis. Since these chromatographic peaks contain glycans that nearly exclusively originate from $\operatorname{IgG}^{28}$, and the IgG glycome was analysed separately, the exclusion of these peaks from the plasma glycome did not lead to any loss of information.

Individual changes of plasma glycome composition in all patients are shown in Figure 2 (average values and their statistical significance are available as Supplementary Table 1). In nearly all analysed individuals glycan groups GP9 (biantennary sialylated structures and oligomannose Man8 structure) and GP16 (tetra-antennary fucosylated structures) increased rapidly after the onset of systemic inflammation, while levels of all other glycan groups decreased. Since we have previously demonstrated that individual plasma glycome has great temporal stability (average $\mathrm{CV}=5.56 \%)^{29}$, the observed changes are apparently related to the disturbance of homeostasis induced by acute inflammation (reproducibility of the analytical procedure is $\mathrm{CV}=7.4 \%)^{29}$.

Glycosylation of IgG. Contrary to the plasma glycome, temporal stability of the IgG glycome within an individual was not examined to date, thus prior to studying changes in IgG glycans after cardiac
Table 1 | Demographic and perioperative data $\mathrm{N}$ 107

\begin{tabular}{lc|}
\hline Age (years) & $67(21-89)$ \\
Male (n/\%) & $81(76)$ \\
BSA (m²) & $2 \pm 0.2$ \\
LVEF (\%) & $54 \pm 13$ \\
Smoking (n/\%) & $14(13)$ \\
Hypertension (n/\%) & $83(78)$ \\
Diabetes (n/\%) & $24(22)$ \\
Chronic pulmonary disease (n/\%) & $9(8)$ \\
Renal failure (n/\%) & $8(7)$ \\
Preoperative medication (n/\%) & \\
Beta blocker & $74(69)$ \\
Statin & $70(65)$ \\
ACE-I & $67(63)$ \\
Operative procedure (n/\%) & \\
Isolated valve surgery & $28(26)$ \\
Isolated coronary bypass surgery & $61(57)$ \\
Combined procedures* & $18(17)$ \\
Perioperative data & \\
Myocardial ischemia (min) & $78 \pm 34$ \\
Cardiopulmonary bypass (min) & $112 \pm 51$ \\
Postoperative stroke (n/\%) & $2(2)$ \\
Perioperative myocardial infarction (n/\%) & $3(3)$ \\
Renal replacement therapy (n/\%) & $3(3)$ \\
30-day mortality (n/\%) & $4(4)$ \\
Highest post-operative CRP median (min-max) & $148(36-399)$ \\
\hline BSA body &
\end{tabular}

BSA, body surface area; LVEF, left ventricular ejection fraction; ACE-I, angiotensin converting enzyme inhibitor.

*Includes multiple valve surgery, valve + coronary surgery, valve + aortic surgery.

surgery, we evaluated "normal" day to day changes by analysing IgG glycosylation in 9 healthy individuals (age 22-78 years) through four days (day 1, day 2 and day 4). Glycans released from IgG molecules were separated into 24 chromatographic peaks (GP1-GP24), individual glycan structures were assigned to each peak and quantified as described previously ${ }^{20}$. A representative chromatogram with glycan structures assigned to each chromatographic peak is shown in Figure 3. The IgG glycome within all individuals was remarkably stable, with average coefficient of variation of 5.9\% (Table 2), what is comparable to variation in repeated measurement of the same sample.

Changes in the IgG glycome composition after cardiac surgery were investigated by analysing $N$-glycans released from isolated IgG (and were thus not affected by changes in the concentration of IgG in plasma). Contrary to the plasma glycome which underwent similar changes in nearly all individuals (Figure 4A), changes in the IgG glycome were very individualized (Figure 4B and Supplementary Figure 1). Despite being discordant between different individuals, these changes were surprisingly extensive and reached over $100 \%$ change for many glycans and individuals (see Supplementary figure 1). In the context of significant intra-individual stability of the IgG glycome composition (Table $2, \mathrm{CV}=5.9 \%$ ), this dramatic change suggests an important role of IgG glycosylation in the studied pathophysiological condition.

Aiming to identify individuals with similar patterns of changes we have applied a bi-clustering algorithm, which in the same time clusters changes in individual glycans and groups individual patients according to similarity in patterns of glycan changes. Four stable groups $(\mathrm{A}-\mathrm{D})$ were identified using this algorithm (Figure 5A). The comparison of these groups with the available clinical data showed that one of the groups has a nearly double average EuroSCORE II (Figure 5B), a composite index used to predict risk of mortality after cardiac surgery ${ }^{30,31}$, that results significantly higher than the other groups $(\mathrm{P}=0.0068,1$-way ANOVA performed with $\log$ (EuroSCORE) values, that have been used due to the nongaussian 


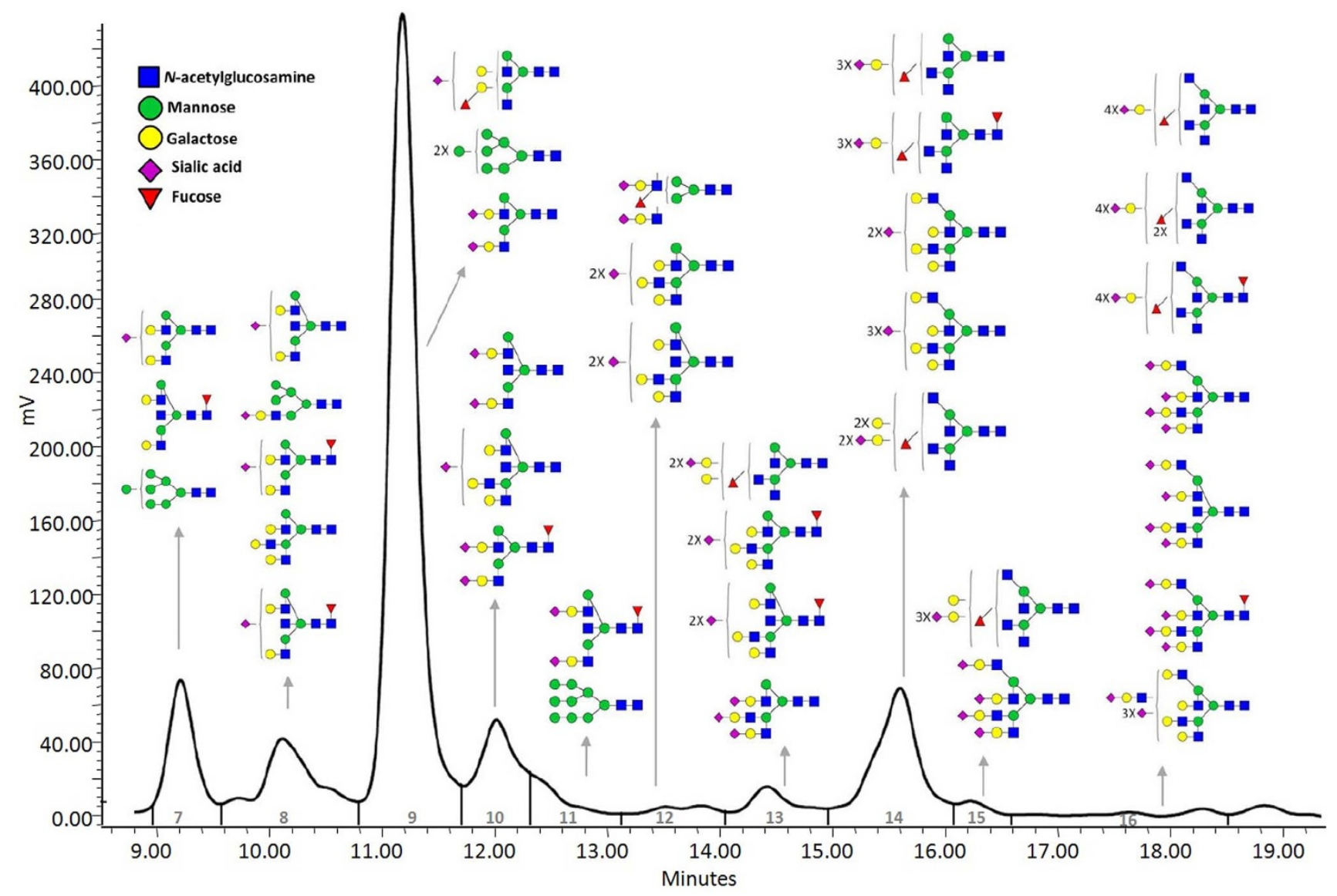

Figure $1 \mid$ Part of the chromatogram of 2-AB labeled N-linked glycans released from plasma proteins and separated by HILIC-HPLC. The integration areas, together with structures present in each glycan group are given. Groups of glycans are numbered from GP7-GP16.

distribution of EuroSCORE values). Interestingly, some clinical variables can be significantly associated with group 2: the number of patients that take diuretic drugs is significantly higher $(P=0.018$, Kruskal-Wallis test), and also a composite index consisting in the number of drugs taken (among 'beta blockers', 'ACE inhibitors', 'diuretics', 'statins', 'amiodaron') is significantly higher in group 2 ( $\mathrm{P}=0.034$, Kruskal-Wallis test). Closer examination of changes in IgG glycans in individual patients from this group (Figure $5 \mathrm{C}$ ) revealed that they are characterized by a rapid decrease in IgG glycans without galactose (GP6: $\mathrm{P}=0.0004$, performed with a coupled Student's T test with a Post-Hoc Bonferroni correction for 96 tests over 24 peaks and 4 groups) and an increase in IgG glycans with two galactoses (GP14, P $=0.0086)$ and glycans with two galactoses and one sialic acid (GP18, P = 0.0067). These changes imply the more rapid increase in the antiinflammatory glycoforms of IgG (galactosylated $^{15}$ and sialylated ${ }^{20,21}$ ) in group 2, than in other groups of patients. Moreover, considering the differences of the values for each peak between day 1 and day 0 , the second peak (GP2) resulted significantly higher in group 2 as compared to the others $(\mathrm{P}=$ 0.023, 1-way ANOVA, see Supplementary figure 2).When we tried to correlate $\log ($ EuroSCORE) with the glycan peaks at different times (Day 0, 1, 3) and with the differences (Day1-Day0, Day3-Day0), some peaks show highly significant correlations/anticorrelations with the score, even if the values are not very high $(|\mathrm{r}| \sim=0.4$ for the highest correlations; Supplementary table 4.).

$\mathrm{N}-$ Glycans as predictors of inflammation severity. When phenotypic characteristics that indicate the rate of the inflammatory response (highest CRP during post-operative hospitalization, number of leukocytes and number of thrombocytes) have been compared to the preoperative levels of main characteristics of IgG glycans previously associated with inflammatory diseases (level of sialylation, fucosylation and presence of bisecting $\mathrm{N}$-acetylglucosamine), statistically significant correlations for fucosylation were observed (Table 3). Statistically significant associations were also observed for the extent of fucosylation change between day 0 and day 1 . Changes in the level of fucosylation can explain over $15 \%\left(\mathrm{R}^{2}=\right.$ 0.15) of the CRP measured, what corresponds to correlation of approximately $\mathrm{r}=0.4$ (Table 3 ). These results indicate that, for example, a patient with high preoperative fucosylation level or moderate fucosylation change would have milder inflammatory response than a patient with low preoperative fucosylation or more prominent change in the level of this glycan feature. Since previously shown connection between glycans and age ${ }^{20}$ suggests that preoperative glycosylation could also be dependent on patient's age, change in fucosylation between day 0 and day 1 has higher clinical potential as prognostic marker. Since opportune intervention is of a great importance the day 0 and day 1 samples are taken into account for the creation of the prognostic marker, whilst the day 3 was neglected.

\section{Discussion}

Cardiac surgery provokes a vigorous inflammatory response with important clinical implications ${ }^{21,22}$, but factors influencing incidence, severity, and clinical outcome of the inflammatory response, and in particular the reason why certain patients develop life-threatening perioperative complications, are currently not well understood. Our recent studies revealed significant inter-individual variation of both total plasma $(\mathrm{CV}=24.03 \%)$ and IgG glycome (CV = 39.4\%) composition ${ }^{19,20}$. Since these values are much higher than those observed for method reproducibility, the inter-individual variation in IgG glycosylation is a consequence of the physiological differences within 

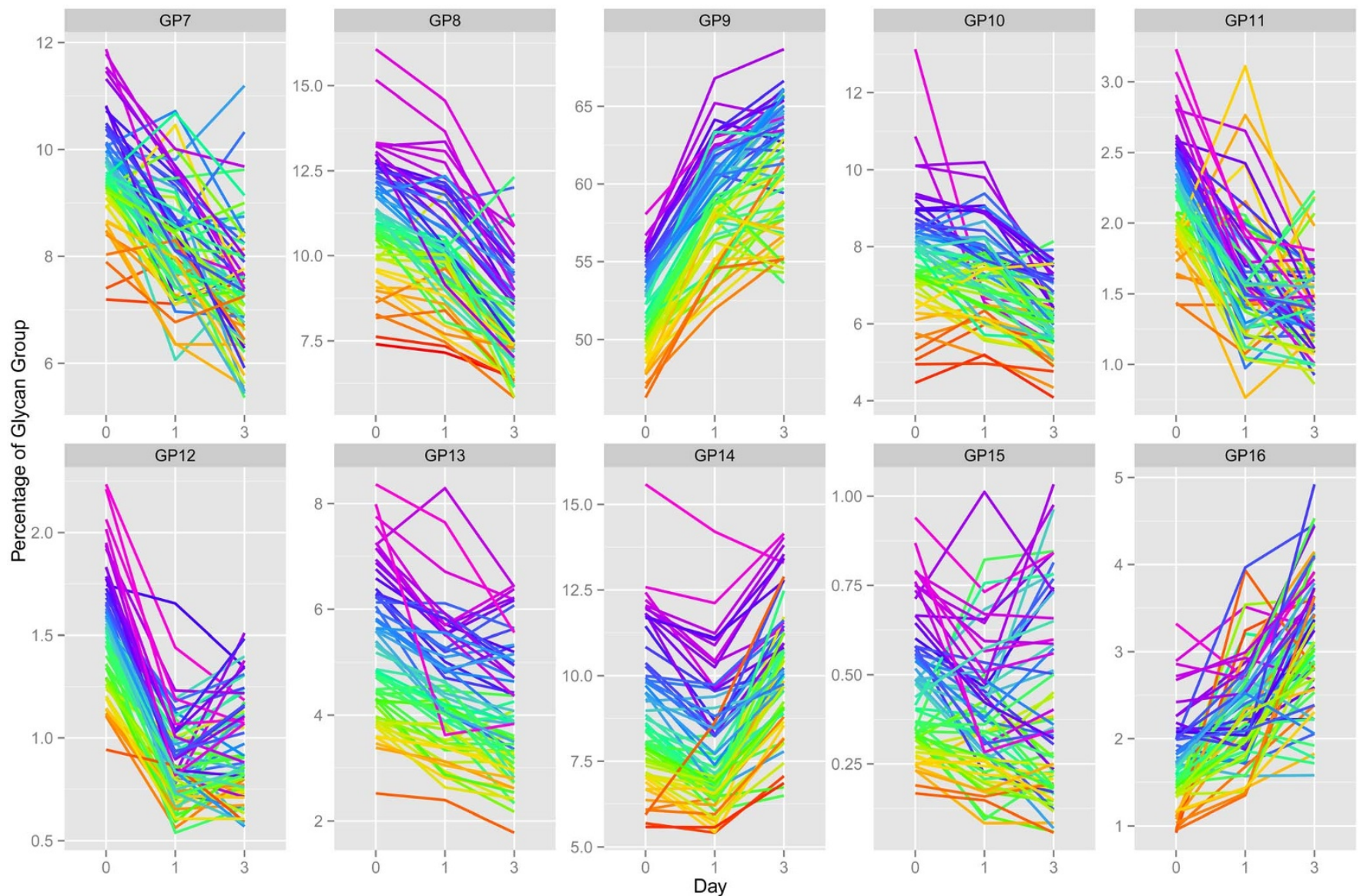

Figure $2 \mid$ Changes in $N$-linked plasma glycans through the initial phase of acute inflammatory response induced by cardiac surgery. Day 0 represents level of glycan group before the surgical procedure, day 1 on the first day after the surgical procedure and day 3 on the third day after the surgery. (The same patient is not represented by the same colour in all graphs, but a same spectrum is used for better visualisation.)

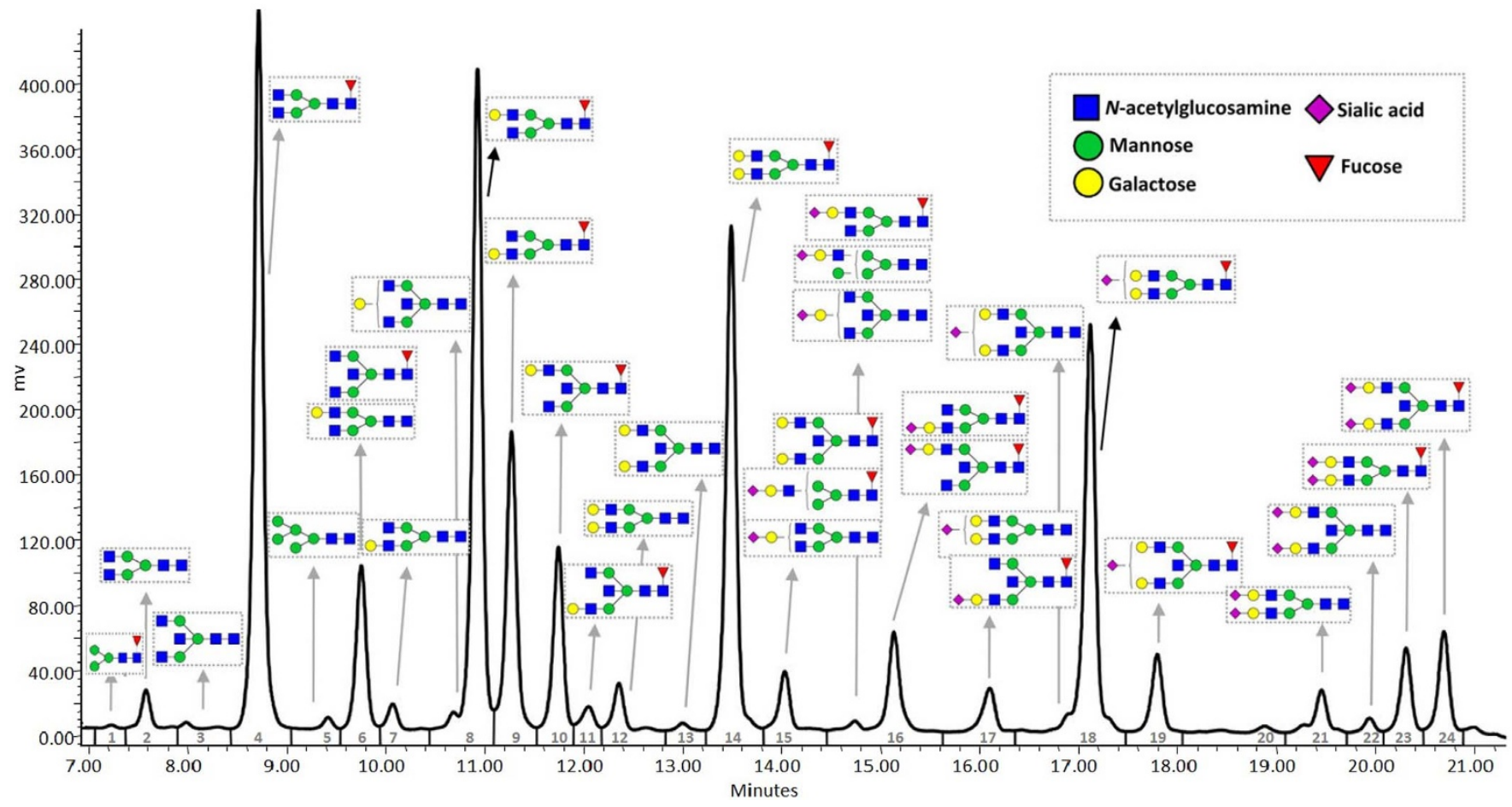

Figure 3 Chromatogram of 2-AB labeled $\mathrm{N}$-linked glycans released from IgG molecules and separated by HILIC-UPLC. The integration areas, together with structures present in each glycan group are given. Groups of glycans are numbered from GP1-GP24, as used in the paper. 
Table 2 | Intra-individual stability of lgG glycans, given as coefficients of variation of all HILC-UPLC separated glycan groups measured through four days in nine individuals

Glycan
group Coefficient of variation (\%)

\begin{tabular}{|c|c|c|c|c|c|c|c|c|c|c|c|}
\hline & Person 1 & Person 2 & Person 3 & Person 4 & Person 5 & Person 6 & Person 7 & Person 8 & Person 9 & average & $\begin{array}{c}\text { reproducibility of } \\
\text { the analytical } \\
\text { method* }\end{array}$ \\
\hline GP1 & 12.6 & 9.1 & 12 & 16.7 & 20 & 0.09 & 9.1 & 8.4 & 14.9 & 11.5 & 26.7 \\
\hline GP2 & 5.06 & 8.6 & 8.9 & 4.56 & 3.6 & 2.77 & 3.8 & 8.1 & 5.22 & 5.62 & 9.97 \\
\hline GP4 & 1.16 & 0.9 & 3.2 & 2.06 & 2.6 & 3.39 & 3.7 & 1.1 & 3.07 & 2.34 & 7.92 \\
\hline GP5 & 7.01 & 2.9 & 21 & 5.52 & 8 & 6.34 & 6.3 & 7.8 & 8.3 & 8.18 & 6.37 \\
\hline GP6 & 0.94 & 1.1 & 4.2 & 0.49 & 3.4 & 1.6 & 3.9 & 1.5 & 2.91 & 2.23 & 11.6 \\
\hline GP9 & 0.45 & 0.4 & 4.7 & 1.78 & 2.1 & 2.01 & 2.6 & 2.6 & 3.48 & 2.23 & 3.06 \\
\hline GP10 & 2.35 & 1.3 & 1.6 & 0.18 & 1.2 & 1.75 & 2.9 & 2.2 & 0.39 & 1.54 & 2.63 \\
\hline GP1 1 & 6.14 & 1.6 & 4.4 & 4.59 & 2.1 & 0.98 & 3.4 & 3.2 & 6.21 & 3.63 & 2.76 \\
\hline GP12 & 1.21 & 2.9 & 13 & 5.06 & 5 & 4.16 & 0.9 & 8.1 & 2.17 & 4.73 & 4.95 \\
\hline GP13 & 6.51 & 5.8 & 15 & 0.97 & 10 & 18.3 & 3.3 & 9.6 & 18.8 & 9.84 & 6.55 \\
\hline GP14 & 1.14 & 0.5 & 1.3 & 1.33 & 1 & 0.81 & 0.7 & 1.3 & 1.67 & 1.08 & 7.58 \\
\hline GP20 & 7.73 & 5 & 4.9 & 15.6 & 9.7 & 10.1 & 30 & 7.3 & 6.5 & 10.7 & 5.08 \\
\hline GP21 & 11.1 & 8.9 & 29 & 13 & 14 & 8.25 & 24 & 13 & 12.7 & 14.8 & 8.54 \\
\hline GP22 & 15.7 & 10 & 21 & 4.56 & 40 & 12.1 & 37 & 21 & 13.4 & 19.4 & 15.9 \\
\hline GP23 & 3.72 & 7.4 & 10 & 0.74 & 5.5 & 3.6 & 26 & 8 & 8.2 & 8.18 & 14.2 \\
\hline GP24 & 5.97 & 8.1 & 8.7 & 0 & 3.6 & 5.58 & 22 & 12 & 5.31 & 7.9 & 9.08 \\
\hline average & 4.78 & 3.76 & 8.25 & 4.18 & 6.56 & 4.37 & 9.12 & 5.81 & 6.53 & 5.93 & 8.65 \\
\hline
\end{tabular}

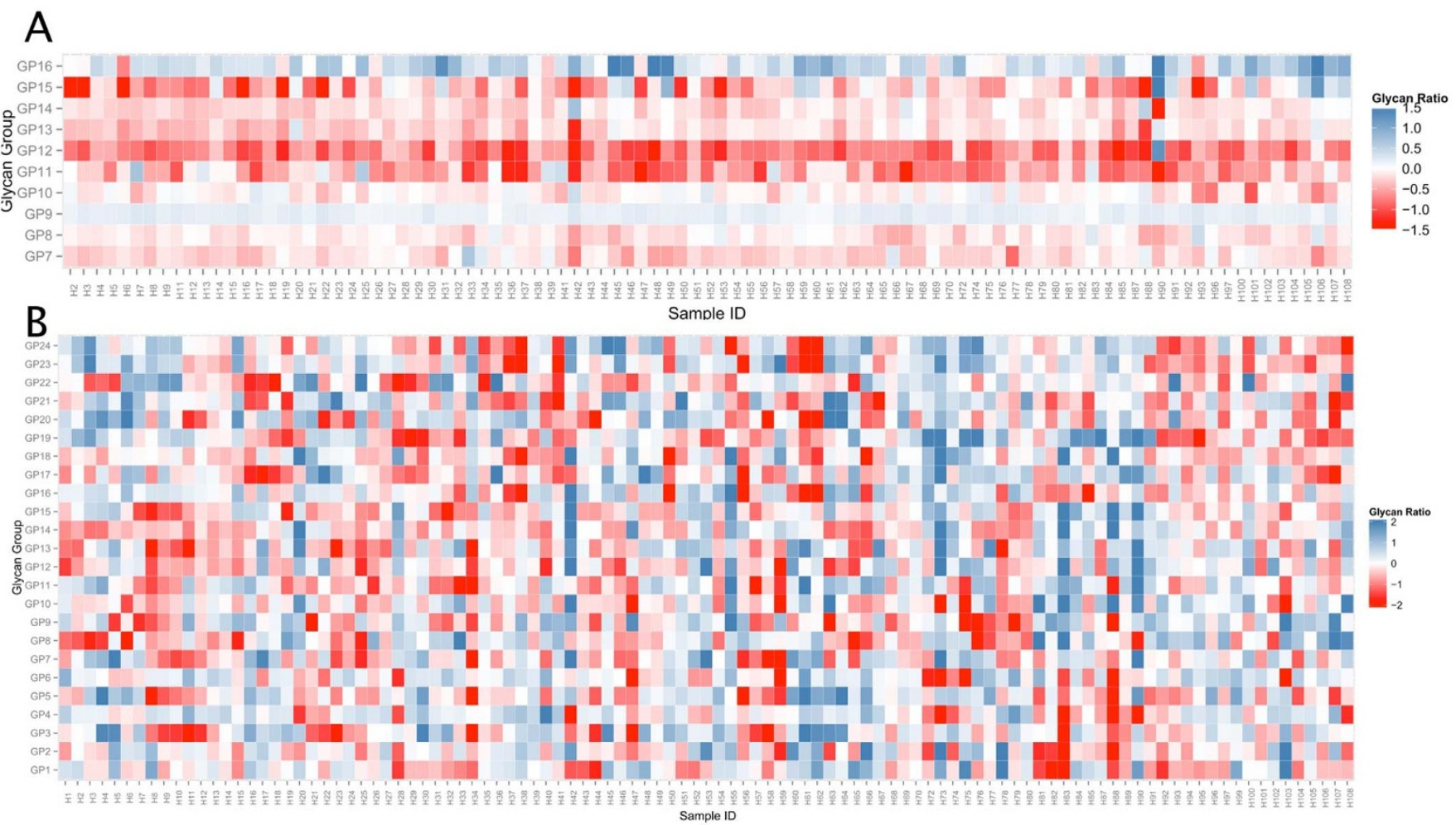

Figure $4 \mid$ Changes in levels of plasma glycans (A) and IgG glycans (B) between day 0 and day 1 . Changes are given as $\log _{2}$ day $1 /$ day 0 . The red colour indicates negative values (decrease in glycan level) and blue colour indicates positive values (increase in glycan level); intensity of the colour indicates the intensity of the change. 

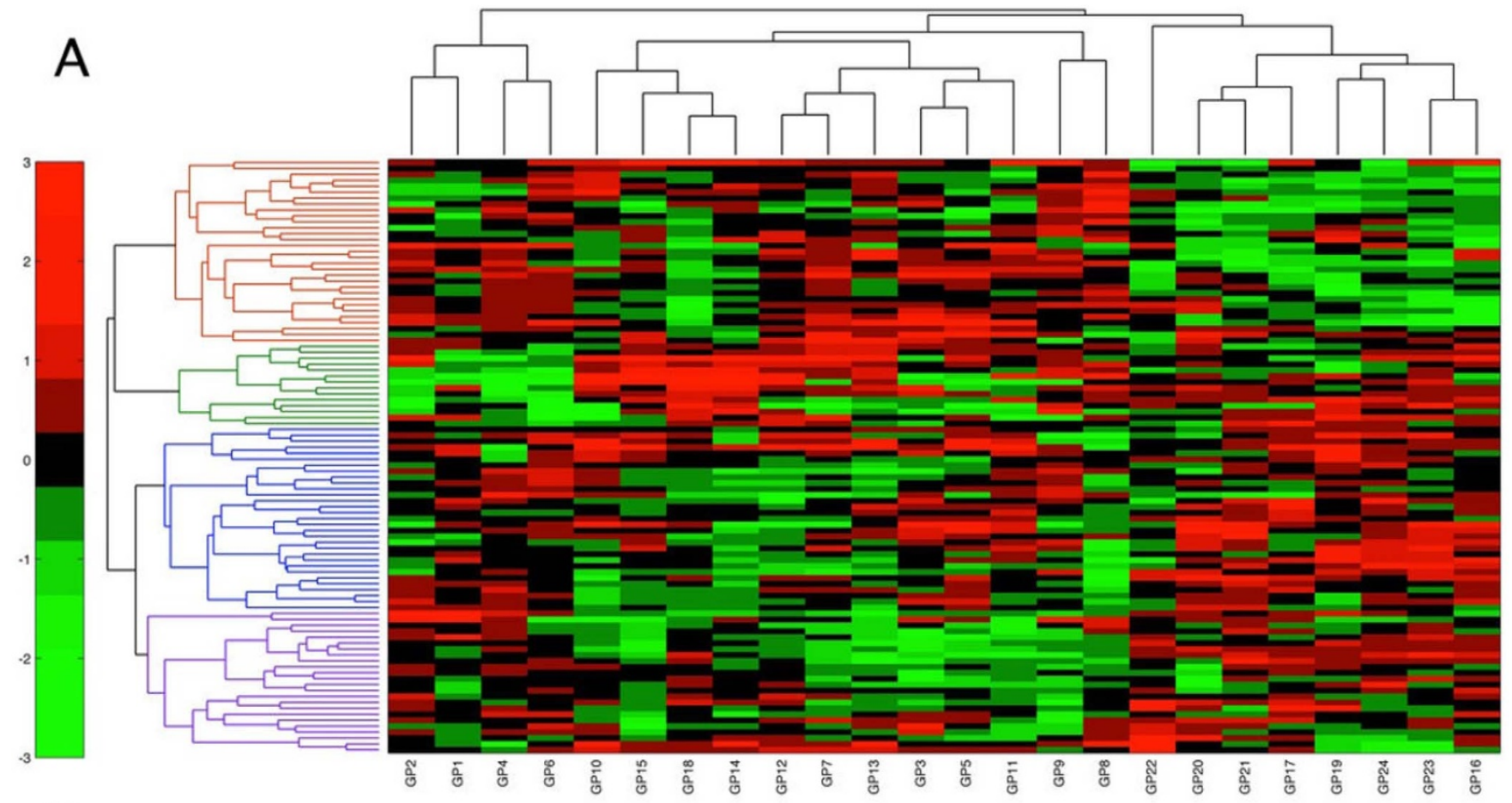

B
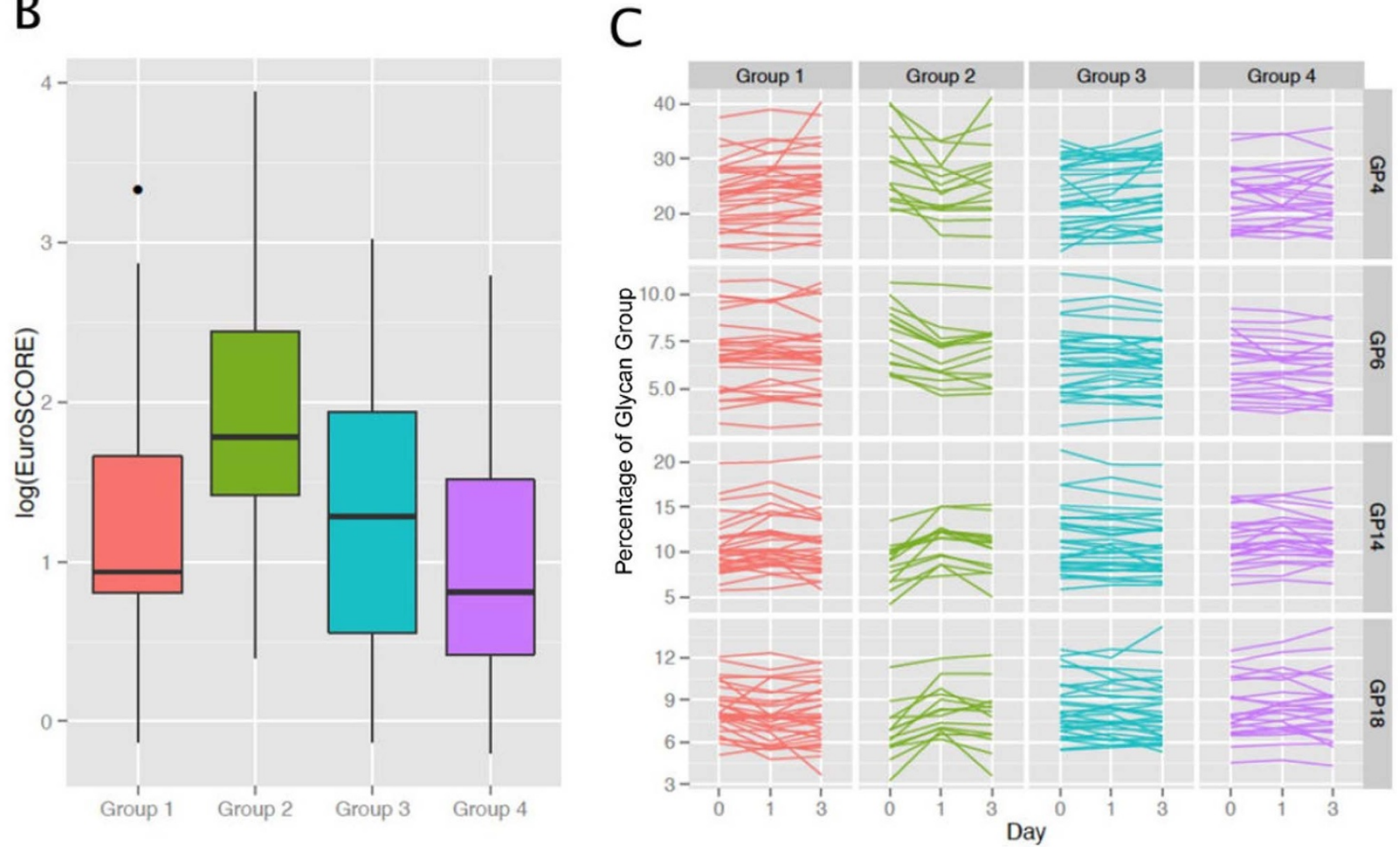

Figure 5 | Bi-clustering analysis of patients and changes in the IgG glycome composition. (A) Bi-clustering heat map; (B) EuroSCORE II in subpopulations generated by bi-clustering; (C) individual changes in IgG glycans in subpopulations generated by bi-clustering.

population rather than limitations of the method. Since glycans have essential structural and functional roles on numerous protein ${ }^{32}$, individual variations in glycosylation are expected to have significant functional implications ${ }^{5,33-35}$. This study is the first large study of individual changes in glycosylation of human IgG and total plasma proteins upon initiation of major inflammation. We used preinflammatory samples from each individual as a baseline control, thus eliminating the problem of the large inter-individual variability in glycosylation that burdens cross-sectional studies. Therefore even though our study was not very large (107 patients), each post-operative sample was matched to a perfect control (pre-operative sample from the same individual).

We observed extensive changes in the glycosylation of plasma proteins after cardiac surgery. Previously we have shown that composition of the plasma glycome is characterized by temporal stability $^{29}$, but it can apparently change very quickly if the homeostasis is 
Table 3 | Connection of glycosylation features related to fucosylation, sialylation and bisecting GlcNAc with measures of severity of inflammatory response (highest CRP, number of leukocytes, number of trombocytes)

\begin{tabular}{|c|c|c|c|c|c|}
\hline Glycan feature & Phenotype & $\mathrm{R}$ & $\mathrm{R}^{2}$ & $p$ & p adjusted \\
\hline FGOn total/GOn & $\max C R P$ & -0.393 & 0.155 & $<0.001$ & 0.003 \\
\hline Fn total & $\max C R P$ & -0.361 & 0.131 & 0.001 & 0.006 \\
\hline FGln total/Gln & $\max C R P$ & -0.336 & 0.113 & 0.002 & 0.010 \\
\hline $\mathrm{FS} / \mathrm{S}$ & $\max C R P$ & -0.277 & 0.077 & 0.010 & 0.042 \\
\hline FG2 $n$ total/G2n & $\max C R P$ & -0.228 & 0.052 & 0.034 & 0.119 \\
\hline$S$ & leukocytes & 0.192 & 0.037 & 0.061 & 0.181 \\
\hline $\mathrm{FS} / \mathrm{S}$ & thrombocytes & 0.084 & 0.007 & 0.423 & 0.888 \\
\hline$\Delta \mathrm{FGOn}$ total/GOn & $\max C R P$ & 0.390 & 0.152 & 0.000 & 0.003 \\
\hline$\Delta \mathrm{FG} / \mathrm{n}$ total/G $\mathrm{n}$ & $\max C R P$ & 0.270 & 0.073 & 0.011 & 0.081 \\
\hline$\Delta \mathrm{FS} / \mathrm{S}$ & $\max C R P$ & 0.264 & 0.070 & 0.014 & 0.081 \\
\hline$\Delta \mathrm{FG} 2 \mathrm{n}$ total/G2n & $\max C R P$ & 0.211 & 0.045 & 0.050 & 0.224 \\
\hline$\Delta S$ & leukocytes & -0.165 & 0.027 & 0.109 & 0.392 \\
\hline
\end{tabular}

FGOn/GOn - the percentage of fucosylation of agalactosylated structures without bisecting GlcNAc;

Fn total - the percentage of all fucosylated structures in total neutral glycan fraction;

FGl $n$ total/Gl $n$ - the percentage of fucosylation of monogalactosylated structures;

FBS total/FS total - ratio of all fucosylated sialylated structures with and without bisecting GlcNAc;

$\mathrm{FS} / \mathrm{S}$ - level of fucosylation of sialylated structures;

FG2 $n$ total/G2 $n$ - the percentage of fucosylation of digalactosylated structures;

$\mathrm{S}$ - the percentage of sialylated structures;

FGOn total/GO - the percentage of fucosylation of agalactosylated structures;

$\Delta$ indicates the difference between day 0 and day 1 .

challenged. Changes in the total plasma protein glycosylation within the first three days after the onset of systemic inflammation followed the same pattern in nearly all patients, indicating the existence of a general physiological response. Differences in protein glycosylation between patients and controls have been previously reported in different inflammatory disorders ${ }^{36}$. Our previous studies revealed changes in glycosylation during the early course of sepsis and acute pancreatitis ${ }^{37-39}$ and the same changes were observed after the acute inflammatory response has been induced in the animal model ${ }^{40}$. However, the design of all previous studies prevented them from identifying very early changes, while in this study we clearly demonstrated very extensive glycosylation changes in the first 24 hours after the onset of inflammation.

The decrease in all sialylated glycans, with the exception of biantennary disialylated structures and tetraantenary fucosylated structures (which increased), occurred within the first 24 hours of inflammation and continued to change in the same direction during the next 48 hours. The notable exceptions from this pattern of changes were triantennary tri sialylated structures with fucose on the outer arm (GP14, also known as the sialyl-Lewis X antigen), which decreased in the first 24 hours, but then significantly increased in the following 48 hours. Though complex and somewhat unexpected, the same pattern was observed in nearly all studied individuals (Figure 2), indicating that this is the real biological phenomenon. Sialyl-Lewis $\mathrm{X}$ antigen has often been associated with inflammation $^{41,42}$ and its structural analogue present on leukocytes is responsible for leukocytes' interaction with selectin ligands on endothelial walls and the initiation of inflammation ${ }^{43-46}$. The increase in this structure on plasma proteins after a rapid decline in the first 24 hours seems to be a reflection of a molecular mechanism that controls inflammation by competitive inhibition of selectin adhesion. However, this part of our study is limited by the fact that plasma glycome composition is affected by both changes in glycosylation and changes in the concentrations of different plasma glycoproteins, thus we cannot distinguish between these two mechanisms.
Contrary to the total plasma glycome, changes in the IgG glycome did not follow a common pattern. Instead, as it is clearly visible in Figure $4 \mathrm{~B}$, IgG glycome composition appears to change nearly at random after the onset of systemic inflammation. However, the extent of these changes in the normally very tightly controlled IgG glycome $e^{47,48}$ is evidently a consequence of specific physiological mechanisms resulting in significantly altered composition of the IgG glycome. Bi-clustering analysis of all patients revealed the existence of four stable groups of patients with distinct patterns of IgG glycosylation changes in the first 24 hours following the cardiac surgery (Figure 5). One of these groups, characterized by a rapid increase in IgG galactosylation, was associated with nearly double EuroSCORE II index. The technology we used to analyse glycans is currently still not routinely used in the clinics, but it is foreseeable that in the near future changes in IgG glycosylation in the first 24 hours after surgery could also be used to identify individuals with higher mortality risk. Furthermore, these individualized changes in IgG glycosylation could also point to molecular mechanisms behind the increased mortality risk. Increased IgG galactosylation has an anti-inflammatory role ${ }^{9}$. The aberrant activation of a wrong glycosylation mechanism (suppression, instead of promotion of inflammation) could be a part of the molecular mechanism leading to increased mortality risk, and thus a potential target for the development of new therapeutic approaches.

Molecular events behind the observed changes in IgG glycosylation are not known, but they could include (i) rapid production of new, alternatively glycosylated molecules of IgG, (ii) specific removal of some IgG glycoforms from circulation, or (iii) specific re-glycosylation in the circulation. IgG half life in plasma is approximately 26 days and since its concentration is plasma is on average $15 \mathrm{~g} / \mathrm{L}$, it seems highly unlikely that a 24 hour period is sufficient for so extensive changes in the glycome through the production of new IgGs. Previous studies of transferrin glycosylation ${ }^{38,40}$ showed its rapid desialylation during inflammation, which can be explained by the activity of specific plasma sialidase or enhanced protein elimination. 
However, the complex glycosylation changes observed on IgG cannot be explained by simple exoglycosidase action. Selective removal of some IgG glycoforms through the interaction of IgG with its receptors and the aggregation of immunoglobulins is a possible mechanism, but another intriguing possibility is the extracellular re-glycosylation of IgG. For decades it is known that numerous glycosyltransferases are specifically released in the circulation, but it was widely believed that concentration of activated sugar nucleotides in plasma is not sufficient for efficient glycosylation. Recently it has been convincingly demonstrated that thrombocytes can release sufficient quantity of activated nucleotides for efficient re-glycosylation at the site of blood clotting ${ }^{49}$. Extracellular sialylation of IgG has also been suggested recently ${ }^{50}$ and the possibility of protein specific extracellular modification of glycosylation should be considered as an option during inflammatory response.

The observed individuality in the IgG glycosylation changes during inflammatory response is also potentially important for the administration of immunoglobulin therapy and could be one of the molecular mechanisms that could explain why this therapeutic approach is efficient in only certain subpopulations of patients. Only a subset of IgG glycoforms are active component of this therapy ${ }^{9,51}$ and highly individualized and extensive glycosylation response is something that has not been evaluated in this context so far.

\section{Methods}

Study population. The institutional ethics committee of the University Hospital Center Zagreb approved the study protocol. All consecutive cardiac surgical patients operated at the University Hospital Center Zagreb from October 2010 to February 2011 were screened for the study. Patients undergoing elective cardiac surgical procedures employing cardiopulmonary bypass met the eligibility criteria. Patients with a left ventricular ejection fraction $<30 \%$, emergency procedures, and those unable to provide consent were excluded from the study. All patients provided written informed consent. 108 patients were enrolled in the study, but full set of samples were collected for a total of 107 patients who were then included in the final analysis. Relevant demographic, clinical and perioperative data are summarized in Table 1. These data were taken from the patients' medical records and were not collected specifically for this study. From all patients blood was drawn on that day, before surgery (day 0 ), and on the first (day 1 ) and third day (day 3 ) following surgery. This period has been chosen due to the essence of the study to observe the early changes during postoperative acute response and to identify potential prognostic glycosylation based markers. The blood was drawn on blood tubes containing EDTA, in the morning, between 7 and 8 a.m. The blood was centrifuged and the plasma was separated and stored at the $-20^{\circ} \mathrm{C}$ until analysis. The study was approved by the University hospital Zagreb Ethical Committee and performed in conformance to the ethical guidelines of the Declaration of Helsinki.

Analysis of $N$-linked plasma glycans. Release of $N$-glycans from plasma proteins. The $5 \mathrm{ml}$ of blood was drawn on the EDTA as an anticoagulant, centrifuged and the plasma was separated. Plasma proteins from $10 \mu \mathrm{l}$ of plasma were reduced by adding dithiotreitol (DTT) (Merck, Darmstadt, Germany) to final concentration of $0.5 \mathrm{M}$, followed by $100 \mathrm{mM}$ iodoacetamide (IAA) (Sigma-Aldrich, St. Louis, MO, USA) alkylation. Denaturated glycoproteins were incorporated in SDS-polyacrylamide gel blocks to minimize the possibility of renaturation and glycoprotein loss. Gels were alternately washed with $100 \%$ acetonitrile (J.T. Baker, Phillipsburg, NJ, USA) and $20 \mathrm{mM} \mathrm{NaHCO}$ (Merck, Darmstadt, Germany) buffer causing continuous dehydration and hydration of the gels, respectively, to remove all free detergents, reducing and alkylating agents. The enzyme, $N$-glycosidase-F (PNGaseF, Prozyme, Hayward, CA, USA) was absorbed by gel blocks and used to release the N-glycans from the proteins. Glycans were removed from gels by alternate washing with water and $100 \%$ acetonitrile, collected in a 96-well collection plate (Waters, Milford, MA, USA) and dried in a vacuum centrifuge (Thermo Scientific, Waltham, MA, USA) ${ }^{52}$

2-AB (2-aminobenzoamide) labeling of released plasma glycans. Dried samples were exposed to $20 \mu \mathrm{L}$ of $1 \%(\mathrm{v} / \mathrm{v})$ formic acid, dried again and finally fluorescently labeled by adding $5 \mu \mathrm{L}$ of $2-\mathrm{AB}$ labeling solution [ $55 \mathrm{mg}$ anthranilamide (Sigma-Aldrich, St. Louis, MO, USA) + 66 mg sodium cyanoborohydride (Sigma-Aldrich, St. Louis, MO, USA) + $330 \mu \mathrm{l}$ glacial acetic acid (Merck, Darmstadt, Germany) $+770 \mu \mathrm{l}$ DMSO (Sigma- Aldrich, St. Louis, MO, USA)]. Mixture was agitated for $10 \mathrm{~min}$, incubated for $30 \mathrm{~min}$ at $65^{\circ} \mathrm{C}$, agitated again for $10 \mathrm{~min}$, and incubated for a further $90 \mathrm{~min}$.

Cleaning and elution of labeled $N$-glycans. In this final step, glycans were immobilized on chromatography paper (GE Healthcare) washed with $100 \%$ acetonitrile to remove the excess of 2-AB and finally collected by elution with $1.5 \mathrm{~mL}$ of water. Samples were completely dried and redissolved in $75 \mu \mathrm{L}$ of water for further analysis.
Hydrophilic Interaction Chromatography (HILIC)-HPLC of plasma glycans. Released glycans were subjected to hydrophilic interaction high performance liquid chromatography (HILIC) on a $250 \mathrm{~mm}$ i.d. $5 \mathrm{~mm}$ particle packed TSKgel Amide 80 column (Tosoh Bioscience, Stuttgart, Germany) at $30^{\circ} \mathrm{C}$ with $50 \mathrm{mM}$ formic acid adjusted to $\mathrm{pH} 4.4$ with ammonia solution as solvent $\mathrm{A}$ and acetonitrile as solvent $\mathrm{B}$. Separation method used linear gradient of $65-53 \%$ acetonitrile $(\mathrm{v} / \mathrm{v})$ at flow rate of $0.8 \mathrm{ml} / \mathrm{min}$ in a $60 \mathrm{~min}$ analytical run. HPLCs were equipped with a Waters temperature control module and a Waters 2475 fluorescence detector set with excitation and emission wavelengths of 330 and $420 \mathrm{~nm}$, respectively. The system was calibrated using an external standard of hydrolyzed and 2-AB-labeled glucose oligomers from which the retention times for the individual glycans were converted to glucose units (GU). Glycans were analyzed on the basis of their elution positions and measured in glucose units then compared to reference values in NIBRT's "GlycoBase v3.0" database available at http://glycobase.nibrt.ie) for structure assignment.

Analysis of IgG glycans. Isolation of IgG from human plasma. The IgG was isolated from the same plasma samples using protein $G$ monolithic plates as described previously ${ }^{20}$. Briefly, $50 \mu \mathrm{l}$ of plasma was diluted 10x with PBS, applied to the protein $\mathrm{G}$ plate and instantly washed. IgGs were eluted with $1 \mathrm{ml}$ of $0.1 \mathrm{M}$ formic acid and neutralized with $1 \mathrm{M}$ ammonium bicarbonate. This method was previously shown to produce the highly purified $\operatorname{IgG}^{19}$ (verified by SDS-PAGE).

$N$-Glycan Release. Isolated IgG samples were dried in a vacuum centrifuge. After drying, proteins were denatured with addition of $20 \mu \mathrm{L} 2 \%$ SDS (w/v) (Invitrogen, Carlsbad, CA, USA) by incubation at $60^{\circ} \mathrm{C}$ for $10 \mathrm{~min}$. Subsequently, $10 \mu \mathrm{L} 4 \%$ Igepal-CA630 (Sigma-Aldrich, St. Louis, MO, USA) and $0.5 \mathrm{mU}$ of PNGase F in $10 \mu \mathrm{L} 5 \times$ PBS were added to the samples. The samples were incubated overnight at $37^{\circ} \mathrm{C}$ for $N$-glycan release.

$2-A B$ labeling. The released $\mathrm{N}$-glycans were subsequently labeled with $2-\mathrm{AB}$. The labeling mixture was freshly prepared by dissolving 2-AB (Sigma-Aldrich, St. Louis, MO, USA) in DMSO (Sigma-Aldrich, St. Louis, MO, USA) and glacial acetic acid (Merck, Darmstadt, Germany) mixture $(17: 3(\mathrm{v} / \mathrm{v}))$ to a final concentration of $48 \mathrm{mg} / \mathrm{mL}$. A volume of $25 \mu \mathrm{L}$ of labeling mixture was added to each $N$-glycan release sample in the 96-well plate. Also, $25 \mu \mathrm{L}$ of freshly prepared reducing agent solution (2-picoline borane (Sigma-Aldrich, St. Louis, MO, USA) in DMSO - concentration of $106.96 \mathrm{mg} / \mathrm{ml}$ ) was added and the plate was sealed using adhesive tape. Mixing was achieved by shaking for $10 \mathrm{~min}$, followed by 2 hour incubation at $65^{\circ} \mathrm{C}$. Samples (in a volume of $100 \mu \mathrm{L}$ ) were brought to $80 \%$ $\mathrm{ACN}(\mathrm{v} / \mathrm{v})$ by adding $400 \mu \mathrm{L}$ of $\mathrm{ACN}$.

Free label and reducing agent were removed from the samples using HILIC-SPE. An amount of $200 \mu \mathrm{L}$ of a $0.1 \mathrm{~g} / \mathrm{mL}$ suspension of microcrystalline cellulose (Merck, Darmstadt, Germany) in water was applied to each well of a $0.45 \mu \mathrm{m}$ GHP filter plate (Pall Corporation, Ann Arbor, MI, USA). Solvent was removed by application of vacuum using a vacuum manifold (Millipore Corporation, Billerica, MA, USA). All wells were prewashed using $5 \times 200 \mu \mathrm{L}$ water, followed by equilibration using $3 \times$ $200 \mu \mathrm{L}$ acetonitrile/water $(80: 20, \mathrm{v} / \mathrm{v})$. The samples were loaded to the wells. The wells were subsequently washed $5 \times$ using $200 \mu \mathrm{L}$ acetonitrile/water $(80: 20, \mathrm{v} / \mathrm{v})$.

Glycans were eluted $2 \times$ with $100 \mu \mathrm{L}$ (instead of $200 \mu \mathrm{L}$ ) of water and combined eluates were not dried, but either analyzed immediately by UPLC or stored at $-20^{\circ} \mathrm{C}$ until usage.

Hydrophilic Interaction Chromatography (HILIC)-UPLC. Fluorescently labeled Nglycans were separated by hydrophilic interaction chromatography on a Waters Acquity UPLC instrument (Milford, MA, USA) consisting of a quaternary solvent manager, sample manager and a FLR fluorescence detector set with excitation and emission wavelengths of 330 and $420 \mathrm{~nm}$, respectively. The instrument was under the control of Empower 2 software, build 2145 (Waters, Milford, MA, USA). Labeled $N$ glycans were separated on a Waters BEH Glycan chromatography column, $100 \times$ $2.1 \mathrm{~mm}$ i.d., $1.7 \mu \mathrm{m} \mathrm{BEH}$ particles, with $100 \mathrm{mM}$ ammonium formate, $\mathrm{pH} 4.4$, as solvent A and acetonitrile as solvent B. Separation method used linear gradient of 75 $62 \%$ acetonitrile $(\mathrm{v} / \mathrm{v})$ at flow rate of $0.4 \mathrm{ml} / \mathrm{min}$ in a $25 \mathrm{~min}$ analytical run. Samples were maintained at $5^{\circ} \mathrm{C}$ before injection, and the separation temperature was $60^{\circ} \mathrm{C}$. The system was calibrated using an external standard of hydrolyzed and 2-AB labeled glucose oligomers from which the retention times for the individual glycans were converted to glucose units. Data processing was performed using an automatic processing method with a traditional integration algorithm after which each chromatogram was manually corrected to maintain the same intervals of integration for all the samples. The chromatograms obtained were all separated in the same manner into 24 peaks and the amount of glycans in each peak was expressed as $\%$ of total integrated area.

Statistical analysis. Batch correction was performed for each glycan group separately using linear mixed model (using programme language $\mathrm{R}$ ). In this model, dependent variable was log transformed fraction of glycan group in total glycome. Age, gender, and time of sampling were described as fixed effects, and individual differences and technical sources of variation were described as random effects. To get corrected values for fractions of glycan groups, estimated batch effect (technical source of variation) was subtracted from each log transformed glycan measurement.

Bi-clustering analysis. Clustering analysis was performed on the difference between IgG glycosylation peak intensity values at day 1 (after surgery) and day 0 (before 
surgery) with the "clustergram" function (Matlab, Mathworks USA) with "average linkage" option and "correlation" distance both for rows (corresponding to each glycosylation peak) and columns (all glycosylation peaks for each sample) defined as one minus Pearson's correlation coefficient (equal to zero for complete correlation and equal to two for complete anticorrelation between rows/columns).

1. Opdenakker, G., Rudd, P. M., Ponting, C. P. \& Dwek, R. A. Concepts and principles of glycobiology. FASEB J 7, 1330-1337 (1993).

2. Moremen, K. W., Tiemeyer, M. \& Nairn, A. V. Vertebrate protein glycosylation: diversity, synthesis and function. Nat Rev Mol Cell Biol 13, 448-462 (2012).

3. Skropeta, D. The effect of individual N-glycans on enzyme activity. Bioorg Med Chem 17, 2645-2653 (2009).

4. Alavi, A. \& Axford, J. S. Sweet and sour: the impact of sugars on disease. Rheumatology (Oxford) 47, 760-770 (2008).

5. Gornik, O., Pavic, T. \& Lauc, G. Alternative glycosylation modulates function of $\mathrm{IgG}$ and other proteins - Implications on evolution and disease. Biochim Biophys Acta 1820, 1318-1326 (2012)

6. Rudd, P. M., Elliott, T., Cresswell, P., Wilson, I. A. \& Dwek, R. A. Glycosylation and the immune system. Science 291, 2370-2376. (2001).

7. Huhn, C., Selman, M. H., Ruhaak, L. R., Deelder, A. M. \& Wuhrer, M. IgG glycosylation analysis. Proteomics 9, 882-913 (2009).

8. Jefferis, R. Glycosylation of recombinant antibody therapeutics. Biotechnol Prog 21, 11-16 (2005)

9. Karsten, C. M. et al. Anti-inflammatory activity of IgG1 mediated by Fc galactosylation and association of FcgammaRIIB and dectin-1. Nat Med (2012).

10. Shields, R. L. et al. Lack of fucose on human IgG1 N-linked oligosaccharide improves binding to human Fcgamma RIII and antibody-dependent cellular toxicity. J Biol Chem 277, 26733-26740 (2002).

11. Ferrara, C., Stuart, F., Sondermann, P., Brunker, P. \& Umana, P. The carbohydrate at FcgammaRIIIa Asn-162. An element required for high affinity binding to nonfucosylated IgG glycoforms. J Biol Chem 281, 5032-5036 (2006).

12. Shinkawa, T. et al. The absence of fucose but not the presence of galactose or bisecting $\mathrm{N}$-acetylglucosamine of human IgG1 complex-type oligosaccharides shows the critical role of enhancing antibody-dependent cellular cytotoxicity. J Biol Chem 278, 3466-3473 (2003).

13. Iida, S. et al. Nonfucosylated therapeutic IgG1 antibody can evade the inhibitory effect of serum immunoglobulin $\mathrm{G}$ on antibody-dependent cellular cytotoxicity through its high binding to FcgammaRIIIa. Clin Cancer Res 12, 2879-2887 (2006).

14. Kaneko, Y., Nimmeriahn, F. \& Ravetch, J. V. Anti-inflammatory activity of immunoglobulin G resulting from Fc sialylation. Science 313, 670-673 (2006).

15. Anthony, R. M. \& Ravetch, J. V. A novel role for the IgG Fc glycan: the antiinflammatory activity of sialylated IgG Fcs. J Clin Immunol 30 Suppl 1, , S9-14 (2010).

16. Anthony, R. M., Wermeling, F., Karlsson, M. C. \& Ravetch, J. V. Identification of a receptor required for the anti-inflammatory activity of IVIG. Proc Natl Acad Sci USA 105, 19571-19578 (2008)

17. Anthony, R. M., Kobayashi, T., Wermeling, F. \& Ravetch, J. V. Intravenous gammaglobulin suppresses inflammation through a novel $\mathrm{T}(\mathrm{H}) 2$ pathway. Nature 475, 110-113 (2011)

18. Anthony, R. M., Nimmerjahn, F., Ashline, D. J., Reinhold, V. N., Paulson, J. C. \& Ravetch, J. V. Recapitulation of IVIG anti-inflammatory activity with a recombinant IgG Fc. Science 320, 373-376 (2008)

19. Knežević, A. et al. Variability, Heritability and Environmental Determinants of Human Plasma N-Glycome. J Proteome Res 8, 694-701 (2009).

20. Pucic, M. et al. High throughput isolation and glycosylation analysis of IgGvariability and heritability of the IgG glycome in three isolated human populations. Mol Cell Proteomics 10, M111 010090 (2011).

21. Raja, S. G. \& Dreyfus, G. D. Modulation of systemic inflammatory response after cardiac surgery. Asian cardiovascular \& thoracic annals 13, 382-395 (2005).

22. Pintar, T. \& Collard, C. D. The systemic inflammatory response to cardiopulmonary bypass. Anesthesiology clinics of North America 21, 453-464 (2003).

23. Moen, O. et al. Attenuation of changes in leukocyte surface markers and complement activation with heparin-coated cardiopulmonary bypass. Ann Thorac Surg 63, 105-111 (1997).

24. de Mendonca-Filho, H. T. et al. Circulating inflammatory mediators and organ dysfunction after cardiovascular surgery with cardiopulmonary bypass: a prospective observational study. Crit Care 10, R46 (2006).

25. Sidebotham, D., McKee, A., Levy, J. \& Gillham, M. Cardiothoracic Critical Care. Elsevier Health Sciences (2007)

26. Hu, D. Management of complex cardiovascular problems: the evidence-based medicine approach. Wiley-Blackwel (2007).

27. Lobato, E. B., Gravenstein, N. \& Kirby, R. R. Complications in anesthesiology. Lippincott Williams \& Wilkins (2007).

28. Bekesova, S. et al. N-glycans in liver-secreted and immunoglogulin-derived protein fractions. Journal of proteomics 75, 2216-2224 (2012).

29. Gornik, O., Wagner, J., Pučić, M., Knežević, A., Redžić, I. \& Lauc, G. Stability of Nglycan profiles in human plasma. Glycobiology 19, 1547-1553 (2009).

30. Durand, E. et al. Performance analysis of EuroSCORE II compared to the original logistic EuroSCORE and STS scores for predicting 30-day mortality after transcatheter aortic valve replacement. The American journal of cardiology 111 891-897 (2013).

31. Roques, F., Michel, P., Goldstone, A. R. \& Nashef, S. A. The logistic EuroSCORE. European heart journal 24, 881-882 (2003).

32. Hart, G. W. \& Copeland, R. J. Glycomics hits the big time. Cell 143, 672-676 (2010).

33. Lauc, G., Vojta, A. \& Zoldos, V. Epigenetic regulation of glycosylation is the quantum mechanics of biology. Biochim Biophys Acta 1840, 65-70 (2013).

34. Lauc, G. \& Zoldoš, V. Protein glycosylation - an evolutionary crossroad between genes and environment. Mol Biosyst 6, 2373-2379 (2010).

35. Zoldos, V., Horvat, T. \& Lauc, G. Glycomics meets genomics, epigenomics and other high throughput omics for system biology studies. Curr Opin Chem Biol 17, 34-40 (2013)

36. Gornik, O. \& Lauc, G. Glycosylation of serum proteins in inflammatory diseases. Dis Markers 25, 267-278 (2008)

37. Gornik, O. et al. Changes of serum glycans during sepsis and acute pancreatitis. Glycobiology 17, 1321-1332 (2007).

38. Gornik, O., Gornik, I., Gasparovic, V. \& Lauc, G. Change in transferrin sialylation is a potential prognostic marker for severity of acute pancreatitis. Clin Biochem 41 , 504-510 (2008)

39. Gornik, O., Gornik, I., Kolednjak, I. Z. \& Lauc, G. Change of transferrin sialylation differs between mild sepsis and severe sepsis and septic shock. Intern Med 50, 861-869 (2011)

40. Piagnerelli, M. et al. Rapid alterations in transferrin sialylation during sepsis. Shock 24, 48-52 (2005).

41. Brinkman-van der Linden, E. C., de Haan, P. F., Havenaar, E. C. \& van Dijk, W. Inflammation-induced expression of sialyl LewisX is not restricted to alpha1-acid glycoprotein but also occurs to a lesser extent on alphal-antichymotrypsin and haptoglobin. Glycoconj J 15, 177-182 (1998).

42. van Dijk, W., Brinkman-Van der Linden, E. C. \& Havenaar, E. C. Occurrence and possible function of inflammation-induced expression of sialyl Lewis-x on acutephase proteins. Adv Exp Med Biol 435, 145-150 (1998).

43. Sperandio, M. Selectins and glycosyltransferases in leukocyte rolling in vivo. FEBS J 273, 4377-4389 (2006).

44. Lowe, J. B. Glycosylation in the control of selectin counter-receptor structure and function. Immunol Rev 186, 19-36 (2002).

45. Mitoma, J. et al. Critical functions of N-glycans in L-selectin-mediated lymphocyte homing and recruitment. Nature Immunology 8, 409-418 (2007)

46. Miyoshi, E., Moriwaki, K. \& Nakagawa, T. Biological function of fucosylation in cancer biology. J Biochem 143, 725-729 (2008).

47. Krištić, J. et al. Glycans are a novel biomarker of chronological and biological age. The journals of gerontology Series A, Biological sciences and medical sciences (2013).

48. Menni, C. et al. Glycosylation of Immunoglobulin G: Role of genetic and epigenetic influences. PLoS One 8, e82558 (2013).

49. Wandall, H. H. et al. The origin and function of platelet glycosyltransferases. Blood (2012).

50. Jones, M. B., Nasirikenari, M., Lugade, A. A., Thanavala, Y. \& Lau, J. T. Antiinflammatory IgG production requires functional P1 promoter in betagalactoside alpha2,6-sialyltransferase 1 (ST6Gal-1) gene. J Biol Chem 287, 15365-15370 (2012).

51. Nimmerjahn, F. \& Ravetch, J. V. Anti-inflammatory actions of intravenous immunoglobulin. Annu Rev Immunol 26, 513-533 (2008).

52. Knezevic, A., Bones, J., Kracun, S. K., Gornik, O., Rudd, P. M. \& Lauc, G. High throughput plasma N-glycome profiling using multiplexed labelling and UPLC with fluorescence detection. The Analyst 136, 4670-4673 (2011).

\section{Acknowledgments}

This work was supported by the European Union's Seventh Framework Programme GlycoBioM (contract \#259869), HighGlycan (contract \#278535), IBD-BIOM (contract \#305479), MIMOmics (contract \#305280) and IntegraLife (contract \#315997) grants. Authors acknowledge the help of medical student Marijeta Pekez in collection of patients' data and thank all examinees for their participation in the study.

\section{Author contributions}

M.N., O.G. and G.L. planned and designed the experiment, E.L., Ž.Đ. and H.G. recruited patients and performed clinical diagnostics, M.N., T.K. and K.R. performed glycan analysis, F.V., D.R., G.C. and O.G. analyzed the data, M.N., O.G. and G.L. wrote the paper

\section{Additional information}

G.L. declares that he is a founder and owner, and M.N. and F.V. declare that they are employees of Genos Ltd, which offers commercial service of glycomic analysis and has several patents in this field. Other authors declare that they have no competing interests. Supplementary information accompanies this paper at http://www.nature.com/ scientificreports

Competing financial interests: The authors declare no competing financial interests. 
How to cite this article: Novokmet, M. et al. Changes in IgG and total plasma protein glycomes in acute systemic inflammation. Sci. Rep. 4, 4347; DOI:10.1038/srep04347 (2014). cc)(i) (2) This work is licensed under a Creative Commons AttributionBY NA SA Nommercial-ShareAlike 3.0 Unported license. To view a copy of this license visit http://creativecommons.org/licenses/by-nc-sa/3.0 